\title{
Perspective: A Standards-Based Manufacturing Information System
}

\author{
Forouzan Golshani* \& Youngchoon Park** \\ * Department of Computer Science and Engineering \\ Arizona State University \\ Tempe, AZ 85287-5406 \\ $+1(602)-965-2855$ \\ ** roz Software Systems, Inc. \\ Scottsdale, AZ 85252-0345
}

\begin{abstract}
We propose an infrastructure and a prototype system for a manufacturing information system. Such a system, by its nature, is distributed and must be able to store, index, manage, retrieve and present data of diverse types, including business data, inventory data, and manufacturing process data. Our approach is different from previous work in that we take into account all possible kinds of information, such as continuous data (stream oriented), production data (decision support), legacy data, and multimedia data (say, drawings, pictures, audio signals, voice annotations, and video streams). The key criterion here for the system is to support content-based information retrieval across all application areas.

The goal is to provide support for automated information transactions in the architecture that uses various standards including STEP (ISO10303) standard, the Internet, and CORBA. An experimental system, called Perspective, for retrieval of part and manufacturing process has been designed and implemented in a distributed environment. Among other capabilities, the system can retrieve a set of parts or manufacturing processes based on similarity to some desired criteria.
\end{abstract}

\section{Keywords}

STEP, Multimedia, Data mining, Content-based Retrieval

The original version of this chapter was revised: The copyright line was incorrect. This has been corrected. The Erratum to this chapter is available at DOI: 10.1007/978-0-387-35390-6_58 


\section{INTRODUCTION}

Rarely, can manufacturing objects, events and processes (i.e., product design process or the fabrication process) be isolated to a single process. Instead, they typically involve a number of complex processes that must be capable of communicating with each other. The global task can be greatly simplified if communications between processes are intelligent and semantically rich. This issue (in the context of a manufacturing enterprise) has been addressed to an extent by the concurrent engineering community (Henderson 1991) (Inmon 1997) (Montana 1993). A concurrent engineering system can be successfully implemented by providing efficient data sharing and collaboration between organizations (or departments) through a set of well-designed collaborative work environments. The rapid development of high performance computer networks (say, ATM Asynchronous Transfer Mode), distributed computing, and distributed database systems will soon allow organizations to share data and collaborate electronically. As a consequence, standards will play an important role in developing of information infrastructure, particularly in the areas of network communications, product modeling, business information and software tools.

A typical information flow in an enterprise is shown in Figure 1. In addition, many standards for information and exchange between enterprises and between departments must be identified and defined.

In this study, we present two things. One is an infrastructure for providing an effective mechanism for information sharing and collaboration. The other is a set of software tools that enable data exchange between various information exchange standards such as STEP and group code.

\section{RELATED TECHNOLOGIES}

\subsection{Standards for Part Coding (ISO10303)}

STEP (ISO10303) is a series of International Standards for the computer-sensible representation and exchange of product data (Gilman 1995) (Industrial Automation Systems 1996). The goal of STEP is to standardize a means of exchanging product data among different computer systems and environments throughout the entire product lifecycle including design, manufacturing, logistic support, repair and disposal. The nature of STEP description makes a product suitable not only for file exchange, but also as a basis for implementing and sharing product databases and archiving. In fact, STEP provides a complete and consistent computer-interpretable product information model that can be used to share product data among the lifecycle phases. STEP has gained widespread industry acceptance. Several CAD system vendors such as $\mathrm{Pro} /$ Engineer ${ }^{\mathrm{TM}}$, UniGraphics ${ }^{\circledR}$, and Computervision are working on STEP AP 203 translator. 


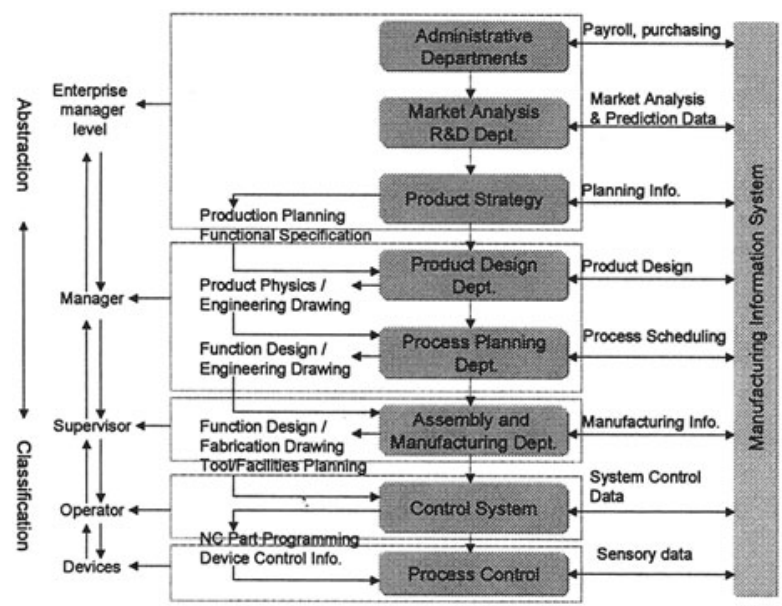

Figure 1. An Enterprise Information Flow Model

Unfortunately, CAD systems have been slow to provide support for database capabilities, and no manufacturing information systems exists that can provide an automatic transaction from CAD systems to manufacturing process management systems. Thus one of our main concerns is the integration of database capabilities with $\mathrm{CAD}$ systems and automatic transaction from $\mathrm{CAD}$ information to manufacturing process management systems.

\subsection{CORBA and Distributed Computing}

An enterprise may have different types of information management system and network connection, and may use different information exchange formats. In such situations, CORBA (Common Object Request Broker Architecture) is a promising technology. An important property of CORBA is the fact that the entire architecture is self-describing. In addition, the specification of a service is always separated from its implementation. This allows for the incorporation of exiting systems into the new and modern developments.

CORBA objects are blobs of intelligence that can reside anywhere on a network. They are packaged as binary components that remote clients can access through method invocations. Both the language and compiler used to create server objects are totally transparent to clients. Clients do not need to know where the distributed object resides or what operating system it executes on. For instance, a server application could be implemented with a COBOL or Fortran, the client does not know the difference. What the client needs to know is the interface of the server object. The specifications of interface are written in a natural Interface Definition Language (IDL). It provides operating system and programming language independent interfaces to all the services and components that reside on a 
CORBA bus. It allows interoperability between client and server objects written in different languages. See Figure 2.

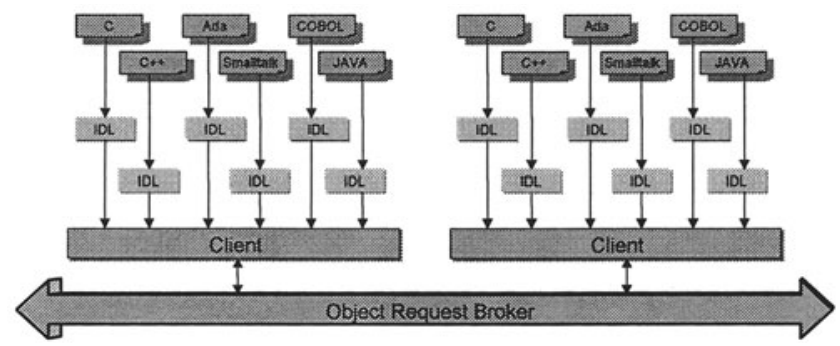

Figure 2. CORBA IDL language building provide Client/Server interoperability

Figure 3 illustrates a distributed computing environment on the WEB. By introducing JAVA, an entirely new client/server application can be developed on the internet. The scenario is as follows. A web client, usually browser (i.e., Netscape Navigator, and Internet Explorer) requests a Java applet. Then the browser initiate a separate TCP/IP session to fetch the applet. The browser loads the applet into the client's main memory, and then executes it. The Java applet can include IDL-generated client stubs, which let it invoke objects on the ORB (Object Request Broker) server. Alternatively, the applet can use the COBRA Dynamic Invocation Interface to generate server request.

Using STEP, and based on the design presented in Figure 3, we can build a computer-aided manufacturing support system. A simplified illustration is presented in Figure 4. (Security issues on the Internet are not yet solved satisfactorily. But this is a more general problem than just manufacturing area.)

\subsection{Group Technology}

One of the major goals of concurrent engineering is to reduce the delivery time.

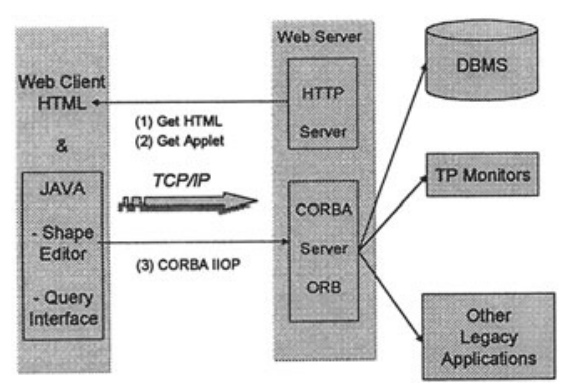

Figure 3. Distributed manufacturing information system on the Internet

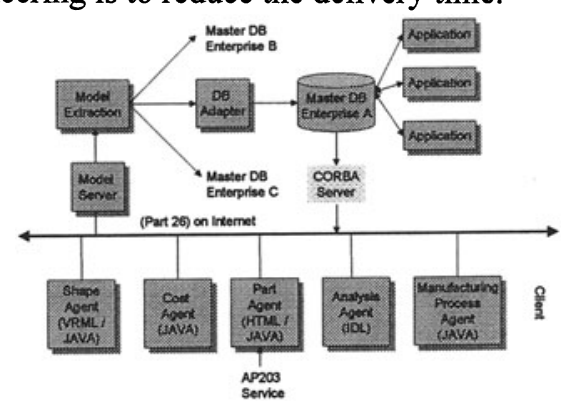

Figure 4. Part and manufacturing data analysis on the internet

Group technology is a method of manufacturing in which parts are classified into groups and appropriate manufacturing processes are defined for each group. It 
provides a means to structure and save information about parts (such as design and manufacturing attributes, processes, and manufacturing capabilities) that is amenable to computerization and analysis. It also provides a common language for the users. Integration of many types of part-related information would be virtually impossible without group technology, particularly when there is a reliance on manufacturing database systems.

\subsection{Part Coding Systems}

Over 100 different coding systems have been developed (Henderson 1991) (Patrick 1993). In this section, we will illustrate STEP (also known as ISO 10303) and two dominant group coding systems (Henderson 1991): DCLASS and MICLASS. DCLASS (Design and Classification Information System) was developed at BYU. It is an eight-digit hybrid code. The DCLASS system has five code segments as shown in Figure 5. The first segment is composed of 3 digits that denote the basic shape of part. The form feature is entered at the forth digit that represents the complexity of a part. The form feature includes shape features such as holes and slots, heat treatments, and special surface finishes. The number of special feature decides the complexity of a part. Other segments indicate the size, precision and types of material.

\begin{tabular}{|l|l|l|l|l|l|l|l|}
\hline 1 & 2 & 3 & 4 & 5 & 6 & 7 & 8 \\
\hline \multicolumn{4}{|c|}{ Basic Shape (3-digit) } & Form & Size & Precision & Material (2-digit) \\
& Feature & &
\end{tabular}

Figure 5. The part code segment of the DCLASS system

Another coding scheme, called MICLASS (Metal Institute Classification) was developed by NOA (Netherlands Organization for Applied Scientific Research). MICLASS system is one of the more popular commercial systems. The MICLASS system represents more detailed information about a part than the DCLASS system. Its major sections are illustrated in Figure 6.

\begin{tabular}{|c|c|c|c|c|c|c|c|c|c|}
\hline 1 & 2 & 3 & 4 & \begin{tabular}{|l|l|}
5 & 6 \\
\end{tabular} & 7 & 8 & \begin{tabular}{l|l} 
& 10 \\
\end{tabular} & 11 & 12 \\
\hline $\begin{array}{l}\text { Main } \\
\text { Shape }\end{array}$ & & lement & $\begin{array}{l}\text { Position } \\
\text { of } \\
\text { shape } \\
\text { element }\end{array}$ & Main dimension & $\begin{array}{l}\text { Dimen- } \\
\text { sion } \\
\text { ratio }\end{array}$ & $\begin{array}{l}\text { Auxili- } \\
\text { ary } \\
\text { dimens- } \\
\text { ion }\end{array}$ & Tolerance code & $\mathrm{Ma}$ & code \\
\hline
\end{tabular}

Figure 6. MICLASS code structure

\subsection{Multimedia Information Systems}

Information required for Decision Support Systems may be kept as various media types such as a newspapers, books, design documents, charts, audio, images and video. Therefore, any integrated information management system must have the capability of multimedia object retrieval and management. The entire manufacturing process and assembly sequence may be produced as moving pictures for the purpose of field engineer training. Many consider multimedia 
information systems to be the most interesting and promising direction to take. Figure 7 shows various media types in the information flow of an enterprise.

\subsection{Data Mining and Data Warehousing Techniques}

A small manufacturing enterprise builds relationships with its customers by taking note of their needs, remembering their preferences, and learning from past interactions how to serve them better in the future. A design engineer designs a part with a specification, identifying and remembering its preferences, and learning from part design heuristic how to design it better in the future. How can a large manufacturing company accomplish something similar when most customers may never interact personally with company employees? How can a design engineer accomplish something similar when other engineers may design most parts? As discussed in a previous section, a set of similar parts may have a set of similar manufacturing processes. In this case, identifying relationships between parts and manufacturing processes are critical.

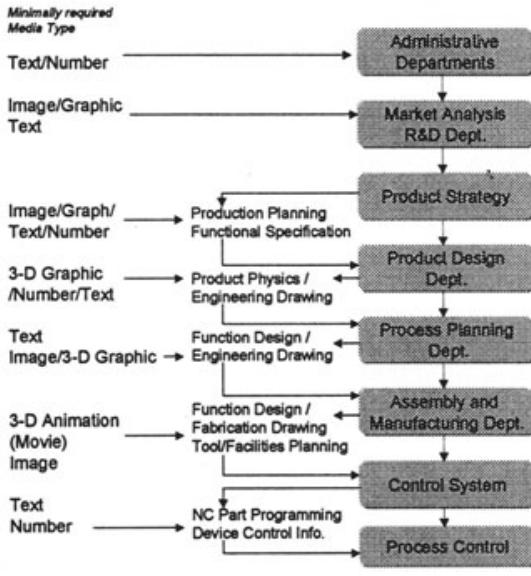

Figure 7. Media types in MIS. (Voice/sound not shown.)

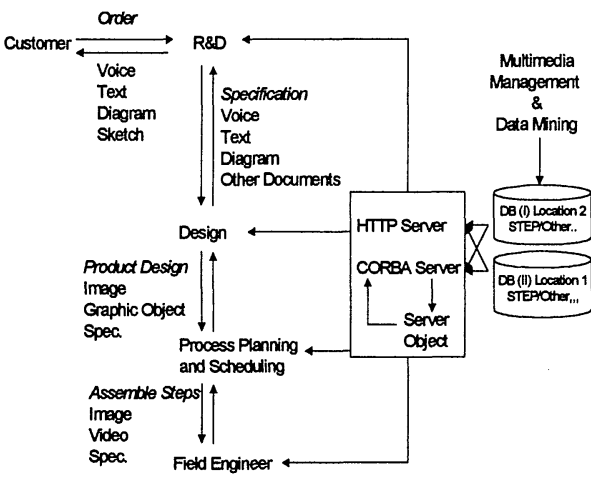

Figure 8. An integrated view

Data mining is the exploration and analysis by automatic or semiautomatic means to discover meaningful patterns and rules. For the purpose of data mining is to allow an enterprise to improve its marketing, sales, and manufacturing design and process. Data mining techniques are well suited for data classification, estimation, prediction, affinity, grouping, clustering and description. In fact, data mining provides the enterprise with intelligence. While the data warehouse provides the enterprise with a memory. Figure 8 illustrates an integrated environment for the multimedia technology based manufacturing information system. 


\section{MANUFACTURING PART DATABASES : A PROTOTYPE}

The complexity of today's products requires that experts from several domains are involved in product design and manufacturing. With the development of computer technologies, a large amount of different software tools and has came into existence in the field of product design and manufacturing. For instance, $\mathrm{CAD} / \mathrm{CAM}$ and CAPP (Computer Aided Process Planning) are widely used in manufacturing.

However, such CAD systems are concerned with geometric information of a product or a part and take no account of design aid facilities, re-use of designer's knowledge and manufacturing database capabilities. In fact, the existing software tools that have been used in the field of manufacturing are restricted to specific task and have difficulties in communicating with one another in order to realize a product that comply with customer demands and that can be manufactured at reasonable cost.

Let us consider the following scenario. A customer wants to have a new hear dryer. The customer gives marketing and R\&D department a textual description and sketch of the hear dryer. Then the marketing and R\&D department will pass a set of information that includes product functions, cost constraints, etc., to designer engineers and manufacturing process engineers. Then a design engineer will face with the task of designing the product would like to know if the same or similar part has been designed before. Similarly, a manufacturing process engineer faced with the task of determining how to manufacture a part would like to know if a similar process plan already exists. Such information flow is shown in Figure 1. We can easily realize the two distinct aspects of the types of information from the scenario. The one is that the information may include pictorial data, graphical objects, textual data, audio and video. In fact, the current manufacturing information can be realized as a multimedia data. The other aspect of the information is related to the data retrieval scheme. The design engineer may need an integrated system that can retrieve a set design-aid information and provides 3$\mathrm{D}$ object design functionality. A collection of desired functionality for the integrated database system is as follows.

- Capability of a part or product recognition. So that, the system will generate a group code such as MICLASS [3] or DCLASS or STEP (STandard for Exchange of Product model data) based part coding. The code can be used to measure the similarity of shape and the similarity of manufacturing process.

- Capability of multimedia object management. Currently, a design document may contain pictorial information, textual data, and others.

- Capability of similarity based query handling and ranked retrieval of retrieval results.

- Hierarchical database navigation mode.

- Capability of design-aided information retrieval based on the similarity. The required query modes are as follows.

- Query by geometric shape; user's query is a CAD object. 
- Query by manufacturing process.

- Query by part/product functionality.

And a manufacturing process engineer may require a system that helps his/her manufacturing process design task. The desired functionality of the system is as follows.

- The system can use a CAD object or group code as input without human assistance.

- The system may have a capability of building user-definable knowledge base that maintains manufacturing process design heuristic and other design-aid knowledge.

As we can see in the list, a capability of similarity-based and contentbased information retrieval is highly demanded to implement an efficient manufacturing information system. Computer technologies can play an important role in manufacturing environments. However, philosophy of computer technologies that support all of manufacturing processes is not available. Some academic systems, which integrate a number of dedicated systems on cooperative basis, are under development such as PACT. Still more research activity is required.

This experimental system deals primarily with support of product design and the link between design and manufacturing by utilizing multimedia information management techniques.

In the following sections, analysis of manufacturing information from the view point of multimedia information management, group technology and a set of feasible computer technologies that enable automated information transaction from design tools to manufacturing information systems are introduced. In addition, the introduced computer technologies may be used in automated manufacturing database cataloging processes.

\subsection{Automated Part Classification and Manufacturing DBMS}

The goal of the automatic part classification is to study of extensions of multimedia database, computer vision, and data mining technologies that enable efficient, interactive exploration of numerous large spatial data sets from within a visualization environment and model based object recognition.

In this study, we have focused on logical and physical system design issues to provide an efficient management of spatial data sets and other information including manufacturing process related data. As a prototype system, named Perspective has been designed. Figure 10 illustrates a computer aided manufacturing environment from the viewpoint of multimedia information management. Figure 10 presents the logical relationship of CAD, CAM and Perspective. 


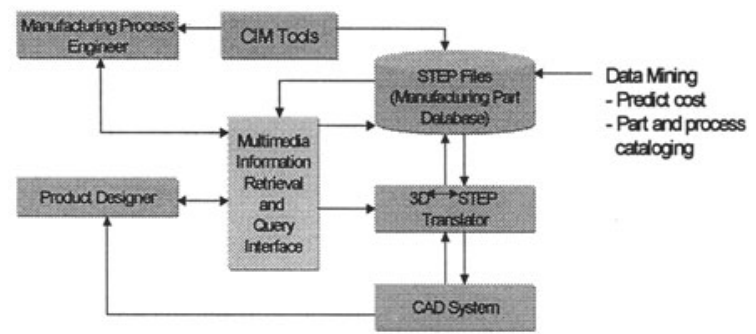

Figure 9. Computer-Aided Manufacturing Environment

Perspective is a database management system that can retrieve a set of three- dimensional objects or a set of manufacturing processes from the database that satisfies the similarity criteria. Its relationships with other technologies are illustrated in Figure 11. Database management capabilities including various query modes are discussed in the following section.

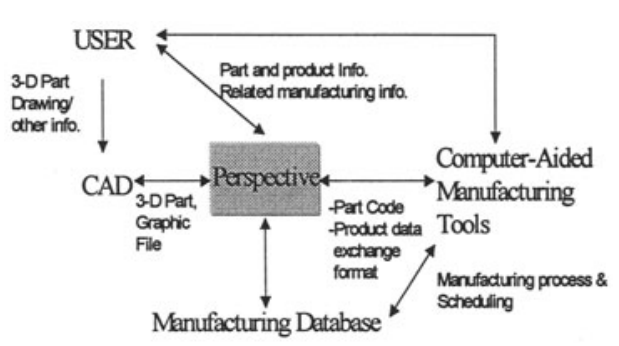

Figure 10. The logical relationship among CAD, CAM and the Perspective

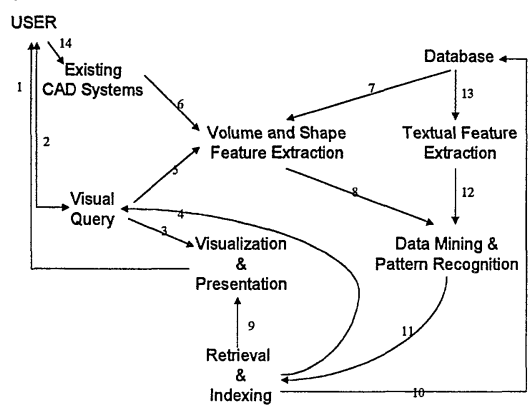

Figure 11. Information flow in Perspective

\subsection{Components and Information Flow in Perspective}

Figure 11 represents the information flows in Perspective. Each numbered arrow indicates a class of information. Numbered-arrows indicate the following:

1. A user receives a set of retrieval results through the visualization and presentation environment - Volume visualization for 3D objects.

2. A user can specify a visual query such as: icon-based similar manufacturing process retrieval, query by shape example, and query by a user-designed object.

3. Visualization/presentation of some of the retrieval results for the user.

4. A set of retrieval results for the given query is provided to visual query. So that, users can refine the query results.

5. If a user provides an own drawing part as a query, the part must be analyzed to extract a set of features for the purpose of matching. 
6. To register a drawing object or part, the part must be analyzed to extract a set of features for the purpose of matching.

7. 3 dimensional parts and products along with documents

8. A set of geometric features.

9. Retrieved data.

10. Retrieval Results.

11. Well-organized part and process classification information.

12. Result of importance-based (biased) textual feature extraction.

13. Actual data.

\subsection{Geometric Features for Part Classification}

Figure 12 represents a set of geometrical features that have been selected for classifying parts in Perspective.

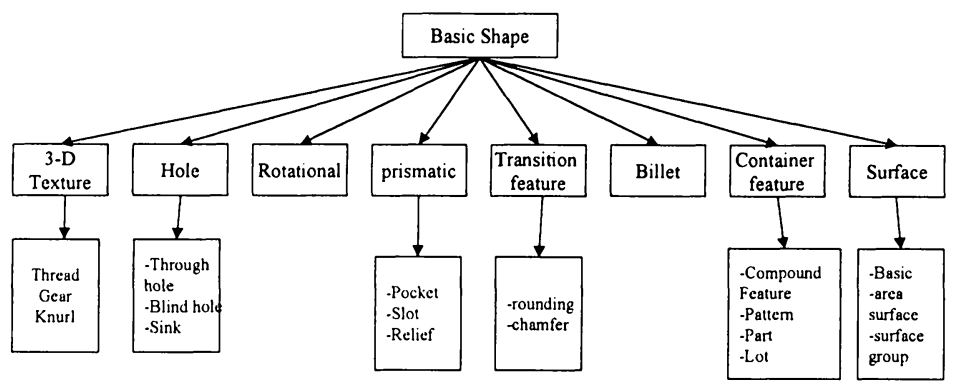

Figure 12. Basic Shape Ontology

Since we are extract the set of geometric features from the CAD object we can easily get the all the information directly from CAD data or by applying simple computations.

\subsection{Similarity-based Part and Process Indexing by Utilizing Data Mining Techniques}

Group technology is based on two rules:

- similar parts have similar part codes

- if two parts have similar part codes, then they require similar manufacturing processes.

As we described earlier, engineering design task and manufacturing process management require similarity-based part and manufacturing process retrieval. The classification task can be accomplished by using data mining techniques. The process of similarity-based part and process indexing is shown in Figure 13. In this demo version, we use Back propagation neural network and GID-3*. 


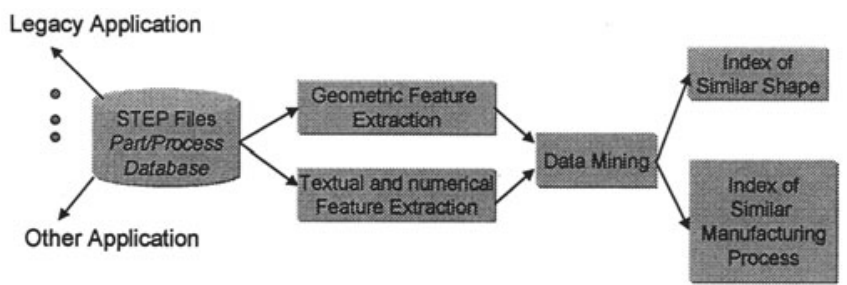

Figure 13. Similarity-based Part and Process Indexing

\subsection{Perspective as a Client Application}

An open, extensible set of protocols for industrial data is essential for the development of distributed manufacturing information systems [8]. The protocols permit enterprises to share industrial data on the Internet and Intranet. These protocols may layered on existing database systems and make it possible for users to process industrial data from any place using low cost WWW.

In this section, we will present a brief explanation of Perspective in distributed environments. A web client that downloads a HTML file to the client, via a browser (i.e., Netscape Navigator, and Internet Explorer) requests a Java applet embodying the Query Interface. Then the browser initiates a separate TCP/IP session to fetch the applet. The browser loads the applet into the client's main memory, and then executes it. The Java applet includes IDL-generated client stubs, which allows invocation of objects on the ORB (Object Request Broker) server. Through this process, users can retrieve similar parts and processes.

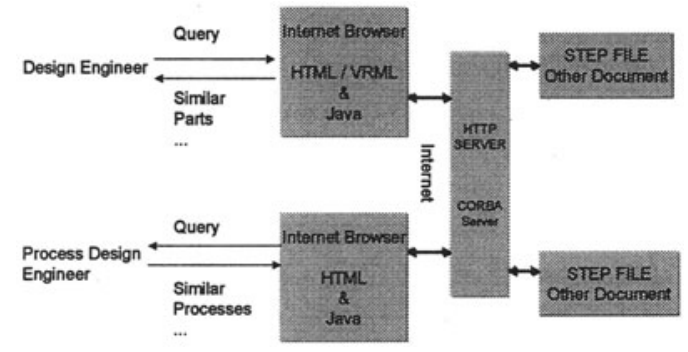

Figure 14. Working environment of Perspective

\section{CONCLUSIONS}

We began our research with the following four goals in mind:

1 Knowledge based support for a multi-level integrated architecture for information flow/ processing : controllers through enterprise

2 Applications of multimedia technologies and visualization in manufacturing

3 Automated analysis and mining of manufacturing data

4 Development of a prototype for some or all of the above. 
To provide an efficient multimedia information sharing and collaboration environments for manufacturing information systems, we present an infrastructure that provides transparent information exchange on the heterogeneous database and network configuration. State-of-art technologies including data mining, contentbased information retrieval, SETP standards and JAVA are involved to implement the architecture.

As an experimental system, named Perspective, that embodies much of the goals set out above, has been developed and tested. It is a web-based part retrieval system incorporating 3D visualization techniques and VRML. Currently, roz Software Systems, (http://www.rozsoftware.com) is handling further development of this system. Future work will focus on enhancing the functionality of the infrastructure and integrating it into a complete collaborative working environment.

\section{REFERENCES}

Mark R. Henderson, David D. Bedworth and Philip M. Wolfe, (1991) ComputerIntegrated Design and Manufacturing, Mc-Graw Hill, 1991.

Blair R. Williams, (1996) Manufacturing for Survival, Addison Wesley,

W. H. Inmon, John A. Zachman, and Jonathan G. Geiger, (1997), Data stores Data Warehousing and The Zachman Framework, Mc-Graw Hill

Patrick J. Montana and Bruce H. Charnov,(1993), Management, $2^{\text {nd }}$ Eds. Barron's Educational Series, Inc.

Charles R. Gilman, and Stephen J. Rock, (1995) "The Use of STEP to integrate Design and Solid Freeform Fabrication", Solid Freeform Fabrication Symposium, U. of Texas at Austin, Austin, TX, August 7-9 .

M. Hardwick, D. Spooner, T. Rando, and K.C Morris, (1996) "Sharing Manufacturing Information in Virtual Enterprises", Communication of $A C M$, Vol. 15, No. 5, Feb.

Industrial Automation Systems, (1996) "Product Data Representation and Exchange : Part 26, IDL Binding for the Standard Data Access Interface," ISO 10303-26, (http://solis.cme.nist.gov), ISO, Geneva, Switzerland.

Usama M. Fayyad Editor, (1996) "Advances in Knowledge Discovery and Data Mining", AAAI Press/The MIT Press.

\section{BIOGRAPHY}

Forouzan Golshani is a Professor of Computer Science and Engineering at Arizona State University (ASU). Previously, he was with the Department of Computing at the Imperial College, London in England. His areas of expertise include multimedia computing, artificial intelligence and database systems.

Youngchoon Park is a Ph.D. student of Computer Science and Engineering at ASU. He received MS degrees from ASU and Chosun University in Korea. His interests include multimedia information retrieval, manufacturing information systems, cluster and group analysis and image and video processing. 